\title{
Infinite sums as linear combinations of polygamma functions
}

\author{
by \\ Kh. Hessami Pilehrood and \\ T. Hessami Pilehrood (Tehran and Shahrekord)
}

Dedicated to Professor Yu. V. Nesterenko on the occasion of his 60th birthday

1. Introduction. 1. We begin with some notations and definitions. Let $d$ be a positive square-free integer. We denote by $\mathbb{Z}, \mathbb{Q}, \overline{\mathbb{Q}}$, and $\mathbb{Q}(i \sqrt{d})$ the set of integers, the field of rational numbers, the field of algebraic numbers, and an imaginary quadratic field, respectively.

We will use the polygamma function

$$
\psi^{(k)}(z)=\frac{d^{k}}{d z^{k}} \psi(z)=\frac{d^{k+1}}{d z^{k+1}} \log \Gamma(z), \quad k=1,2, \ldots,
$$

which has the following series expansion (see $[2, \S 1.16]$ ):

$$
\psi^{(k)}(z)=(-1)^{k+1} k ! \sum_{n=0}^{\infty} \frac{1}{(n+z)^{k+1}}, \quad z \neq 0,-1,-2, \ldots,
$$

and the logarithmic derivative of $\Gamma(z)$,

$$
\psi(z)=\frac{d}{d z} \log \Gamma(z)=-\gamma+\sum_{n=0}^{\infty}\left(\frac{1}{n+1}-\frac{1}{n+z}\right), \quad z \neq 0,-1,-2, \ldots,
$$

called the digamma function. Obviously, $\psi(1)=-\gamma$, where $\gamma$ is Euler's constant. The function $\psi^{(k)}(z), k=0,1,2, \ldots$, is single-valued and analytic in the whole complex plane except for the points $z=-m, m=0,1,2, \ldots$,

2000 Mathematics Subject Classification: Primary 11J81.

Key words and phrases: polygamma function, digamma function, transcendental number, Schanuel conjecture.

Research of Kh. Hessami Pilehrood supported in part by a grant from IPM (No. 85110020).

Research of T. Hessami Pilehrood supported in part by a grant from IPM (No. $85110021)$. 
where it has poles of order $k+1$. The polygamma function satisfies many functional relations $[2, \S 1.16]$ such as

- "recurrence formula":

$$
\psi^{(k)}(z+1)=\psi^{(k)}(z)+\frac{(-1)^{k} k !}{z^{k+1}},
$$

- "reflection formula":

$$
\psi^{(k)}(1-z)+(-1)^{k+1} \psi^{(k)}(z)=(-1)^{k} \pi \frac{d^{k}}{d z^{k}} \cot \pi z,
$$

- "multiplication formula":

$$
\psi^{(k)}(m z)=\delta \log m+\frac{1}{m^{k+1}} \sum_{r=0}^{m-1} \psi^{(k)}\left(z+\frac{r}{m}\right),
$$

where $\delta=1$ if $k=0$ and $\delta=0$ if $k>0$.

We also introduce its alternating analog (see $[2, \S 1.16]$ )

$$
g^{(k)}(z)=(-1)^{k} k ! \sum_{n=0}^{\infty} \frac{(-1)^{n}}{(n+z)^{k+1}}=\frac{1}{2^{k+1}}\left(\psi^{(k)}\left(\frac{z+1}{2}\right)-\psi^{(k)}\left(\frac{z}{2}\right)\right),
$$

which satisfies the similar functional relations

$$
\begin{gathered}
g^{(k)}(z+1)=\frac{(-1)^{k} k !}{z^{k+1}}-g^{(k)}(z), \quad k=1,2, \ldots \\
g^{(k)}(z)+(-1)^{k} g^{(k)}(1-z)=\pi \frac{d^{k}}{d z^{k}}\left(\frac{1}{\sin \pi z}\right) .
\end{gathered}
$$

Obviously by (1) and (4), the numbers $\psi^{(k)}(1) / \zeta(k+1), g^{(k)}(1) / \zeta(k+1)$, $\psi^{(k)}(1 / 2) / \zeta(k+1)$ are rational (here $\zeta(s)=\sum_{n=1}^{\infty} 1 / n^{s}$ is the Riemann zeta function) and therefore from (2), (5) we get the following inclusions:

$$
\psi^{(2 k-1)}(m), g^{(2 k-1)}(m), \psi^{(2 k-1)}(m+1 / 2) \in \mathbb{Q}^{\times} \cdot \pi^{2 k}+\mathbb{Q}, \quad m \in \mathbb{N} .
$$

2. In this paper, we consider the values of the series

$$
S=\sum_{n=0}^{\infty} \frac{P(n)}{Q(n)}, \quad T=\sum_{n=0}^{\infty} \frac{P(n)}{Q(n)}(-1)^{n}, \quad U=\sum_{n=0}^{\infty} \frac{P(n)}{Q(n)} f(n),
$$

where $P(x), Q(x) \in \overline{\mathbb{Q}}[x]$ and $f$ is a periodic number-theoretic function, and express them as linear combinations of values of the polygamma functions (see Lemmas 1-2 below). Such a representation allows one to give simple sufficient conditions for the numbers $S, T$ to be algebraic or transcendental, which is done in Section 2. Further, we assume that all the zeros of $Q(x)$ are in the imaginary quadratic field $\mathbb{Q}(i \sqrt{d})$ and the polynomials $P(x), Q(x)$ have some symmetry properties. By formulas (3), (6), summing the series 
$S, T, U$ explicitly and applying Nesterenko's famous result [7] on algebraic independence of the numbers $\pi, e^{\pi \sqrt{d}}$ we show that the infinite sums (8) either have a computable algebraic value or are transcendental. (By a computable value, we mean a number which can be explicitly determined in terms of its defining parameters.) Actually, we describe a mixed approach for computation of infinite sums (8) combining linear combinations of values of the polygamma functions and contour integration. The latter can be applied to the trigonometric series

$$
V=\sum_{n=-\infty}^{\infty} \frac{P_{1}(n) e^{i \beta_{1} n}+\cdots+P_{s}(n) e^{i \beta_{s} n}}{Q(n)}, \quad \beta_{1}, \ldots, \beta_{s} \in \mathbb{Q},
$$

and enables us to prove that under certain conditions on the polynomials $P_{1}, \ldots, P_{s}, Q$, the sum $V$ is either zero or transcendental. As a consequence, we establish the transcendence of some Fourier series (see Section 4). In Section 5 we extend these results to a more general set of roots of the polynomial $Q(x)$ provided that the Schanuel conjecture holds. This generalizes the well-known result of $\mathrm{P}$. Bundschuh on the series $\sum_{n=2}^{\infty} 1 /\left(n^{2 k}-1\right), k \geq 2$ (see [3], [12, Section 3.2]).

Special cases of the infinite sums (8) were considered by P. Bundschuh in [3]. Using Baker's theory on linear forms in logarithms, he proved that the value of the series

$$
F(z)=z \sum_{m=1}^{\infty} \frac{a_{m}}{m(m-z)},
$$

where $\left\{a_{m}\right\}_{m=1}^{\infty}$ is a periodic sequence of algebraic numbers and $z \in \mathbb{Q} \cap$ $(0,1)$, is either zero or transcendental. In particular, this yields the transcendence of the numbers $\psi(z)+\gamma, \psi(z)-\psi(z / 2)$ for any $z \in \mathbb{Q} \backslash \mathbb{Z}$, and of the series $\sum_{n=2}^{\infty} \zeta(n) z^{n}, \sum_{n=2}^{\infty} \beta(n) z^{n}$ for any rational $z$ with $0<|z|<1$, where $\beta(s)=\sum_{k=0}^{\infty}(-1)^{k} /(2 k+1)^{s}$ is the Dirichlet beta function.

The case when all the roots $\alpha_{1}, \ldots, \alpha_{m}$ of $Q(x)$ are distinct rational numbers was considered in [1], where by Baker's theory it was proved that each of the numbers (8) is either a computable algebraic number or is transcendental. In particular, if $Q(x)$ is a reduced polynomial, i.e., if $\alpha_{1}, \ldots, \alpha_{m}$ are distinct rational numbers from $[-1,0)$, then $S, T, U$ and the series

$$
\sum_{n=0}^{\infty} \frac{P_{1}(n) \beta_{1}^{n}+\cdots+P_{s}(n) \beta_{s}^{n}}{Q(n)}, \quad \beta_{1}, \ldots, \beta_{s} \in \overline{\mathbb{Q}},
$$

are either zero or transcendental.

Notice that from [1] it follows that for any rational numbers $\alpha_{1}, \ldots, \alpha_{m}$ distinct from nonnegative integers and such that $\alpha_{k}-\alpha_{l} \notin \mathbb{Z}, 1 \leq k \neq l \leq m$, 
all the values

$$
\psi\left(\alpha_{1}\right), \ldots, \psi\left(\alpha_{m}\right)
$$

are transcendental except for at most one value of $\alpha_{k}$ (compare this with [6, Theorem 3]). In fact, taking into account (2) we can assume without loss of generality that $\alpha_{1}, \ldots, \alpha_{m}$ are distinct numbers from $(0,1]$ and then by $[1$, Theorem 3] we have, for $k \neq l$,

$$
\psi\left(\alpha_{l}\right)-\psi\left(\alpha_{k}\right)=\sum_{n=0}^{\infty}\left(\frac{1}{n+\alpha_{k}}-\frac{1}{n+\alpha_{l}}\right)=\sum_{n=0}^{\infty} \frac{\alpha_{l}-\alpha_{k}}{\left(n+\alpha_{k}\right)\left(n+\alpha_{l}\right)} \notin \overline{\mathbb{Q}} .
$$

Therefore the set (9) cannot contain two algebraic numbers.

In 2001, G. Molteni [5] considered the generating power series for the sequence $\{\zeta(2 k+1)\}_{k=1}^{\infty}$, which can also be written as a linear combination of values of the digamma function,

$$
F(z)=\sum_{k=1}^{\infty} \zeta(2 k+1) z^{2 k}=-\frac{1}{2} \psi(1+z)-\frac{1}{2} \psi(1-z)+\psi(1),
$$

and proved that the numbers $1, F\left(\alpha_{1}\right), \ldots, F\left(\alpha_{m}\right)$ are linearly independent over $\overline{\mathbb{Q}}$ if all $\alpha_{k}=a_{k} / b_{k}$ are distinct rational numbers from the interval $(0,1)$ such that $\left(a_{k}, b_{k}\right)=1$ and for any $k$ there exists an odd prime $p_{k}$ dividing $b_{k}$ and $p_{k} \nmid b_{j}$ when $j \neq k$. An obvious corollary is that $F(\alpha)$ is transcendental for all $\alpha=a / b \in(0,1)$ with $b$ not a power of 2 . Actually, this restriction can be removed and $F(\alpha)$ is transcendental for any rational $\alpha$ with $0<|\alpha|<1$ by [1, Theorem 3], since

$$
F(\alpha)=\sum_{n=0}^{\infty} \frac{\alpha^{2}}{(n+1)(n+1+\alpha)(n+1-\alpha)}
$$

and the last series does not vanish.

\section{Sums $S, T, U$ as linear combinations of polygamma functions}

LEMma 1. Let $f: \mathbb{Z} \rightarrow \overline{\mathbb{Q}}$ be periodic with period $q \in \mathbb{N}$. Suppose that $P(x), Q(x) \in \overline{\mathbb{Q}}[x], \operatorname{deg} P(x) \leq \operatorname{deg} Q(x)-1$, and $Q(x)=\left(x+\alpha_{1}\right)^{l_{1}} \ldots$ $\ldots\left(x+\alpha_{m}\right)^{l_{m}}$, where $l_{1}, \ldots, l_{m} \in \mathbb{N}$ and $\alpha_{1}, \ldots, \alpha_{m}$ are distinct, and distinct from non-negative integers. If $\operatorname{deg} P(x)=\operatorname{deg} Q(x)-1$, suppose also that $\sum_{t=0}^{q-1} f(t)=0$ (convergence condition). Then the series

$$
U=\sum_{n=0}^{\infty} \frac{P(n)}{Q(n)} f(n)
$$

converges and we have the following representation:

$$
U=\sum_{t=0}^{q-1} f(t) \sum_{k=1}^{m} \sum_{l=1}^{l_{k}} \frac{(-1)^{l}}{(l-1) !} \frac{A_{k, l}}{q^{l}} \psi^{(l-1)}\left(\frac{t+\alpha_{k}}{q}\right)
$$


with

$$
A_{k, l}=\left.\frac{1}{\left(l_{k}-l\right) !} \frac{d^{l_{k}-l}}{d x^{l_{k}-l}}\left(\frac{P(x)}{Q(x)}\left(x+\alpha_{k}\right)^{l_{k}}\right)\right|_{x=-\alpha_{k}} \in \overline{\mathbb{Q}} .
$$

Proof. Writing $n$ in the form $n=q \tau+t, \tau, t \in \mathbb{Z}, 0 \leq t \leq q-1, \tau \geq 0$, we get

$$
U=\sum_{\tau=0}^{\infty} \sum_{t=0}^{q-1} f(q \tau+t) \frac{P(q \tau+t)}{Q(q \tau+t)}=\sum_{\tau=0}^{\infty} \sum_{t=0}^{q-1} f(t) \frac{P(q \tau+t)}{Q(q \tau+t)}
$$

Decomposing $P(x) / Q(x)$ into partial fractions, we have

$$
\frac{P(x)}{Q(x)}=\sum_{k=1}^{m} \sum_{l=1}^{l_{k}} \frac{A_{k, l}}{\left(x+\alpha_{k}\right)^{l}}
$$

where the coefficients $A_{k, l}$ are defined in (11) and $\sum_{k=1}^{m} A_{k, 1}=0$ if $\operatorname{deg} P(x) \leq \operatorname{deg} Q(x)-2$.

To prove (10), we first suppose that $\operatorname{deg} P(x) \leq \operatorname{deg} Q(x)-2$. Then from (12) we have

$$
U=\sum_{t=0}^{q-1} f(t) \sum_{\tau=0}^{\infty} \frac{P(q \tau+t)}{Q(q \tau+t)}
$$

where

$$
\begin{aligned}
\frac{P(q \tau+t)}{Q(q \tau+t)} & =\sum_{k=1}^{m} \sum_{l=1}^{l_{k}} \frac{A_{k, l}}{\left(q \tau+t+\alpha_{k}\right)^{l}} \\
& =\sum_{k=1}^{m} \frac{A_{k, 1}}{q \tau+t+\alpha_{k}}+\sum_{k=1}^{m} \sum_{l=2}^{l_{k}} \frac{A_{k, l}}{\left(q \tau+t+\alpha_{k}\right)^{l}} \\
& =\frac{1}{q} \sum_{k=2}^{m} A_{k, 1}\left(\frac{1}{\tau+\frac{t+\alpha_{k}}{q}}-\frac{1}{\tau+\frac{t+\alpha_{1}}{q}}\right)+\sum_{k=1}^{m} \sum_{l=2}^{l_{k}} \frac{A_{k, l}}{\left(q \tau+t+\alpha_{k}\right)^{l}} .
\end{aligned}
$$

Therefore,

$$
\begin{aligned}
\sum_{\tau=0}^{\infty} \frac{P(q \tau+t)}{Q(q \tau+t)}= & \frac{1}{q} \sum_{k=2}^{m} A_{k, 1}\left(\psi\left(\frac{t+\alpha_{1}}{q}\right)-\psi\left(\frac{t+\alpha_{k}}{q}\right)\right) \\
& +\sum_{k=1}^{m} \sum_{l=2}^{l_{k}} \frac{(-1)^{l}}{(l-1) !} \frac{A_{k, l}}{q^{l}} \psi^{(l-1)}\left(\frac{t+\alpha_{k}}{q}\right) \\
= & \sum_{k=1}^{m} \sum_{l=1}^{l_{k}} \frac{(-1)^{l}}{(l-1) !} \frac{A_{k, l}}{q^{l}} \psi^{(l-1)}\left(\frac{t+\alpha_{k}}{q}\right),
\end{aligned}
$$


which yields (10). If $\operatorname{deg} P(x)=\operatorname{deg} Q(x)-1$, then we find

$$
\begin{aligned}
& \sum_{t=0}^{q-1} \frac{P(q \tau+t)}{Q(q \tau+t)} f(t)=\sum_{t=0}^{q-1} f(t) \sum_{k=1}^{m} \sum_{l=1}^{l_{k}} \frac{A_{k, l}}{\left(q \tau+t+\alpha_{k}\right)^{l}} \\
& =\sum_{t=0}^{q-1} f(t) \sum_{k=1}^{m} \frac{A_{k, 1}}{q \tau+t+\alpha_{k}}+\sum_{t=0}^{q-1} f(t) \sum_{k=1}^{m} \sum_{l=2}^{l_{k}} \frac{A_{k, l}}{\left(q \tau+t+\alpha_{k}\right)^{l}} \\
& =\sum_{k=1}^{m} \frac{A_{k, 1}}{q} \sum_{t=1}^{q-1} f(t)\left(\frac{1}{\tau+\frac{t+\alpha_{k}}{q}}-\frac{1}{\tau+\frac{\alpha_{k}}{q}}\right)+\sum_{t=0}^{q-1} f(t) \sum_{k=1}^{m} \sum_{l=2}^{l_{k}} \frac{A_{k, l}}{\left(q \tau+t+\alpha_{k}\right)^{l}} .
\end{aligned}
$$

Hence, by (12), we get

$$
\begin{aligned}
U=\sum_{k=1}^{m} & \frac{A_{k, 1}}{q} \sum_{t=1}^{q-1} f(t)\left(\psi\left(\frac{\alpha_{k}}{q}\right)-\psi\left(\frac{t+\alpha_{k}}{q}\right)\right)+\sum_{t=0}^{q-1} f(t) \sum_{k=1}^{m} \sum_{l=2}^{l_{k}} \frac{(-1)^{l}}{(l-1) !} \frac{A_{k, l}}{q^{l}} \\
\times \psi^{(l-1)}\left(\frac{t+\alpha_{k}}{q}\right) & =\sum_{t=0}^{q-1} f(t) \sum_{k=1}^{m} \sum_{l=1}^{l_{k}} \frac{(-1)^{l}}{(l-1) !} \frac{A_{k, l}}{q^{l}} \psi^{(l-1)}\left(\frac{t+\alpha_{k}}{q}\right),
\end{aligned}
$$

as required.

Let us mention two particular cases $q=1, f \equiv 1$ and $q=2, f(n)=(-1)^{n}$ of Lemma 1.

Lemma 2. Let $P(x), Q(x) \in \overline{\mathbb{Q}}[x], Q(x)=\left(x+\alpha_{1}\right)^{l_{1}} \ldots\left(x+\alpha_{m}\right)^{l_{m}}$, where $l_{1}, \ldots, l_{m} \in \mathbb{N}$ and $\alpha_{1}, \ldots, \alpha_{m}$ are distinct, and distinct from non-negative integers. Suppose that the series

$$
S=\sum_{n=0}^{\infty} \frac{P(n)}{Q(n)}, \quad T=\sum_{n=0}^{\infty} \frac{P(n)}{Q(n)}(-1)^{n}
$$

converge. Then the following representations are valid:

$$
S=\sum_{k=1}^{m} \sum_{l=1}^{l_{k}} \frac{(-1)^{l}}{(l-1) !} A_{k, l} \psi^{(l-1)}\left(\alpha_{k}\right), \quad T=\sum_{k=1}^{m} \sum_{l=1}^{l_{k}} \frac{(-1)^{l-1}}{(l-1) !} A_{k, l} g^{(l-1)}\left(\alpha_{k}\right),
$$

where the coefficients $A_{k, l}$ are defined in (11).

If $Q(x)$ has only simple zeros, then Lemma 2 enables us to give simple sufficient conditions for $S, T$ to be algebraic or transcendental.

Corollary 1. Let $P(x), Q(x) \in \overline{\mathbb{Q}}[x], Q(x)=\left(x+\alpha_{1}\right) \ldots\left(x+\alpha_{m}\right)$, where $\alpha_{1}, \ldots, \alpha_{m}$ are distinct, and distinct from non-negative integers, and $\operatorname{deg} P(x) \leq \operatorname{deg} Q(x)-2$. If there is a subset $L$ of $\{1, \ldots, m\}$ with $\# L \geq 2$, with $j, k \in L \Rightarrow \alpha_{j}-\alpha_{k} \in \mathbb{Z}$, and with $P\left(-\alpha_{l}\right)=0$ for $l \notin L$, then

$$
S=\sum_{n=0}^{\infty} \frac{P(n)}{Q(n)}
$$

is algebraic. 
Proof. This statement easily follows from Lemma 2 and formula (2).

Remark 0.1. In the case $m=3$ and $\alpha_{1}, \ldots, \alpha_{m} \in \mathbb{Q}, P(x), Q(x) \in \mathbb{Q}[x]$ the conditions of Corollary 1 are necessary and sufficient for $S$ to be rational (see [9, Theorem 2]).

Corollary 2. Let $P(x), Q(x) \in \overline{\mathbb{Q}}[x], Q(x)=\left(x+\alpha_{1}\right) \ldots\left(x+\alpha_{m}\right)$, where $\alpha_{1}, \ldots, \alpha_{m}$ are distinct, and distinct from non-negative integers, and $\operatorname{deg} P(x) \leq \operatorname{deg} Q(x)-1$. If $\alpha_{k}-\alpha_{1}=: n_{k} \in \mathbb{Z}$ for all $1 \leq k \leq m$ and

$$
\sum_{k=1}^{m}(-1)^{n_{k}} \frac{P\left(-\alpha_{k}\right)}{Q^{\prime}\left(-\alpha_{k}\right)}=0
$$

then

$$
T=\sum_{n=0}^{\infty} \frac{P(n)}{Q(n)}(-1)^{n}
$$

is algebraic. (In particular, if all $n_{k}$ are even and $\operatorname{deg} P(x) \leq \operatorname{deg} Q(x)-2$, then condition (13) holds automatically.)

Proof. This statement easily follows from Lemma 2 and formula (5).

Remark 0.2. In the case $m=2$ and $\alpha_{1}, \ldots, \alpha_{m} \in \mathbb{Q}, P(x), Q(x) \in \mathbb{Q}[x]$ the conditions of Corollary 2 are necessary and sufficient for $T$ to be rational (see [9, Theorem 1] and [10, Theorem 3]).

Corollary 3. Let $P(x) \in \overline{\mathbb{Q}}[x], Q(x)=\left(x+\alpha_{1}\right) \ldots\left(x+\alpha_{m}\right)$, where $\alpha_{1}, \ldots, \alpha_{m}$ are distinct rational numbers, distinct from non-negative integers, and $\operatorname{deg} P(x)=m-1$. If $\alpha_{k}-\alpha_{l} \in 2 \mathbb{Z}$ for all $1 \leq k, l \leq m$, then the sum

$$
T=\sum_{n=0}^{\infty} \frac{P(n)}{Q(n)}(-1)^{n}
$$

is transcendental.

Proof. By Lemma 2 and formula (5) it follows that

$$
T=A+a g\left(\alpha_{1}\right)=B \pm a g(\alpha)=B \pm a \sum_{n=0}^{\infty}\left(\frac{1}{2 n+\alpha}-\frac{1}{2 n+\alpha+1}\right),
$$

where $A, B \in \overline{\mathbb{Q}}, a \neq 0$ is the leading coefficient of the polynomial $P(x)$ and $\alpha \equiv \alpha_{1}(\bmod 1), \alpha \in(0,1]$. Since the infinite sum in the latter expression of $T$ does not vanish, by $[1$, Theorem 3$]$ we conclude that $T$ is transcendental.

Lemma 3. For the $k$ th derivatives we have

$$
\text { (a) }(\cot \pi z)^{(k)}=\pi^{k} p_{k}(\cot \pi z), \quad \text { (b) }\left(\frac{1}{\sin \pi z}\right)^{(k)}=\pi^{k} \frac{q_{k}(\cos \pi z)}{\sin ^{k+1} \pi z},
$$

where $p_{k}(z), q_{k}(z) \in \mathbb{Z}[z], \operatorname{deg}\left(p_{k}(z)-(-1)^{k} k ! z^{k+1}\right) \leq k, \operatorname{deg}\left(q_{k}(z)-(-z)^{k}\right)$ $\leq k-1$. 
Proof. The proof is by induction on $k$. Obviously, for $k=0$ formulas (a), (b) are valid with $p_{0}(z)=z$ and $q_{0}(z)=1$. Assuming (a), (b) to hold for $k$, we will prove them for $k+1$. We have

$$
(\cot \pi z)^{(k+1)}=\pi^{k}\left(p_{k}(\cot \pi z)\right)^{\prime}=\pi^{k+1} p_{k+1}(\cot \pi z),
$$

where $p_{k+1}(z)=-p_{k}^{\prime}(z)\left(z^{2}+1\right)=(-1)^{k+1}(k+1) ! z^{k+2}+c_{k+1} z^{k+1}+\cdots \in$ $\mathbb{Z}[z]$, and

$$
\left(\frac{1}{\sin \pi z}\right)^{(k+1)}=\pi^{k}\left(\frac{q_{k}(\cos \pi z)}{\sin ^{k+1} \pi z}\right)^{\prime}=\pi^{k+1} \frac{q_{k+1}(\cos \pi z)}{\sin ^{k+2} \pi z}
$$

with $q_{k+1}(z)=q_{k}^{\prime}(z)\left(z^{2}-1\right)-(k+1) z q_{k}(z)=(-1)^{k+1} z^{k+1}+d_{k} z^{k}+\cdots \in$ $\mathbb{Z}[z]$.

\section{Main results}

Theorem 1. Let $P_{1}, \ldots, P_{s}, Q_{1}, \ldots, Q_{s} \in \overline{\mathbb{Q}}[x], m_{1}, \ldots, m_{s} \in \mathbb{N}, r_{1}, \ldots, r_{s}$ $\in \mathbb{Z}$ satisfy the following conditions: for any $1 \leq j \leq s, \operatorname{deg} P_{j} \leq \operatorname{deg} Q_{j}-2$,

$$
\frac{P_{j}(-x)}{Q_{j}(-x)}=\frac{P_{j}\left(r_{j}+x\right)}{Q_{j}\left(r_{j}+x\right)}
$$

$Q_{j}(x)=\prod_{k=1}^{2 m_{j}}\left(x-\alpha_{j, k}\right)^{l_{j, k}}$, where $\alpha_{j, k}=a_{j, k}+i b_{j, k} \sqrt{d} \in \mathbb{Q}(i \sqrt{d}) \backslash \mathbb{N}_{0}$, $k=1, \ldots, 2 m_{j}$, are distinct and such that $\alpha_{j, m_{j}+k}=r_{j}-\alpha_{j, k}, b_{j, k} \geq 0$, $l_{j, m_{j}+k}=l_{j, k} \in \mathbb{N}, k=1, \ldots, m_{j}$. Then the sum

$$
S=\sum_{n=0}^{\infty}\left(\frac{P_{1}(n)}{Q_{1}(n)}+\cdots+\frac{P_{s}(n)}{Q_{s}(n)}\right)
$$

is either a computable algebraic number or transcendental. Moreover, $S$ is transcendental if at least one of the following conditions holds:

(i) $\alpha_{j, k} \notin \mathbb{Q} \backslash \mathbb{Z}, j=1, \ldots, s, k=1, \ldots, 2 m_{j}$, and

$$
\sum_{\substack{j=1 \\ \alpha_{j, k} \notin \mathbb{Z}}}^{s} \sum_{\substack{m_{j} \\ z=\alpha_{j, k}}} \frac{P_{j}(z)}{Q_{j}(z)} \neq 0,
$$

(ii) $b_{j_{0}, k_{0}}:=\min \left\{b_{j, k}: b_{j, k}>0\right\}$ is a unique minimum of the positive numbers $b_{j, k}$ and $\operatorname{res}_{z=\alpha_{j_{0}, k_{0}}} P_{j_{0}}(z) / Q_{j_{0}}(z) \neq 0$,

(iii) there exists a unique maximum $l_{j_{0}, k_{0}}$ of the sequence $l_{j, k}, 1 \leq j \leq s$, $1 \leq k \leq m_{j}$, and $b_{j_{0}, k_{0}}>0, P_{j_{0}}\left(\alpha_{j_{0}, k_{0}}\right) \neq 0$.

Proof. By Lemma 2, we have

$$
S=\sum_{j=1}^{s} \sum_{n=0}^{\infty} \frac{P_{j}(n)}{Q_{j}(n)}=\sum_{j=1}^{s} \sum_{k=1}^{2 m_{j}} \sum_{l=1}^{l_{j, k}} \frac{(-1)^{l}}{(l-1) !} A_{j, k, l} \psi^{(l-1)}\left(-\alpha_{j, k}\right),
$$


where

$$
A_{j, k, l}=\left.\frac{1}{\left(l_{j, k}-l\right) !}\left(\frac{d}{d x}\right)^{l_{j, k}-l}\left(\frac{P_{j}(x)}{Q_{j}(x)}\left(x-\alpha_{j, k}\right)^{l_{j, k}}\right)\right|_{x=\alpha_{j, k}} \in \overline{\mathbb{Q}} .
$$

From (14), (15) for $1 \leq k \leq m_{j}$ it follows that

$$
\begin{aligned}
A_{j, m_{j}+k, l} & =\left.\frac{1}{\left(l_{j, k}-l\right) !}\left(\frac{d}{d x}\right)^{l_{j, k}-l}\left(\frac{P_{j}\left(r_{j}-x\right)}{Q_{j}\left(r_{j}-x\right)}\left(x-r_{j}+\alpha_{j, k}\right)^{l_{j, k}}\right)\right|_{x=r_{j}-\alpha_{j, k}} \\
& =\left.\frac{(-1)^{l}}{\left(l_{j, k}-l\right) !}\left(\frac{d}{d y}\right)^{l_{j, k}-l}\left(\frac{P_{j}(y)}{Q_{j}(y)}\left(y-\alpha_{j, k}\right)^{l_{j, k}}\right)\right|_{y=\alpha_{j, k}}=(-1)^{l} A_{j, k, l}
\end{aligned}
$$

with $y=r_{j}-x$. Therefore,

$$
S=\sum_{j=1}^{s} \sum_{k=1}^{m_{j}} \sum_{l=1}^{l_{j, k}} \frac{(-1)^{l}}{(l-1) !} A_{j, k, l}\left(\psi^{(l-1)}\left(-\alpha_{j, k}\right)+(-1)^{l} \psi^{(l-1)}\left(\alpha_{j, k}-r_{j}\right)\right) .
$$

Now if for some pair $(j, k)$ we have $-\alpha_{j, k}$ and $\alpha_{j, k}-r_{j} \in \mathbb{N}$, then by (2), (7), we get

$$
\begin{aligned}
& S=C_{0}+\sum_{j=1}^{s} \sum_{k=1}^{m_{j}} \sum_{l=1}^{l_{j, k}} C_{j, k, l} \pi^{l} \\
& \alpha_{j, k} \in \mathbb{Z} \quad l \text { even } \\
& +\sum_{j=1}^{s} \sum_{k=1}^{m_{j}} \sum_{l=1}^{l_{j, k}} \frac{A_{j, k, l}}{(l-1) !}\left(\psi^{(l-1)}\left(\alpha_{j, k}+1\right)+(-1)^{l} \psi^{(l-1)}\left(-\alpha_{j, k}\right)\right), \\
& \alpha_{j, k} \notin \mathbb{Z}
\end{aligned}
$$

where $C_{0}, C_{j, k, l} \in \overline{\mathbb{Q}}$. Combining this with (3) and Lemma 3 we conclude that

$$
\begin{aligned}
& S=C_{0}+\sum_{\substack{j=1 \\
\alpha_{j, k} \in \mathbb{Z}}}^{s} \sum_{\substack{l=1 \\
l \text { even }}}^{m_{j}} \sum_{\substack{l=1 \\
l_{j, k}}} C_{j, k, l} \pi^{l} \\
& +\sum_{j=1}^{s} \sum_{k=1}^{m_{j}} \sum_{l=1}^{l_{j, k}} \frac{(-1)^{l-1} A_{j, k, l}}{(l-1) !} \pi^{l} p_{l-1}\left(-\cot \pi \alpha_{j, k}\right) . \\
& \alpha_{j, k} \notin \mathbb{Z}
\end{aligned}
$$

According to the formula

$$
\cot \pi \alpha_{j, k}=i \frac{e^{2 \pi i a_{j, k}}+e^{2 \pi b_{j, k} \sqrt{d}}}{e^{2 \pi i a_{j, k}}-e^{2 \pi b_{j, k} \sqrt{d}}}=-i-\frac{2 i e^{2 \pi i a_{j, k}}}{e^{2 \pi b_{j, k} \sqrt{d}}-e^{2 \pi i a_{j, k}}}
$$

we see that $S-C_{0} \in \overline{\mathbb{Q}}\left(\pi, e^{\pi \sqrt{d} / B}\right)$, where $B \in \mathbb{N}$ is the least common denominator of the numbers $b_{j, k}$, and therefore $S-C_{0}$ is either zero or transcendental in view of the algebraic independence of $\pi$ and $e^{\pi \sqrt{d}}[7]$. 
If we suppose that $S$ is algebraic and condition (i) holds, then considering the summands in (16) involving $\pi$ to the first power we get

$$
-\pi \sum_{\substack{j=1 \\ \alpha_{j, k} \notin \mathbb{Z}}}^{s} \sum_{\substack{k=1 \\ m_{j}}} A_{j, k, 1} \cot \pi \alpha_{j, k}+\pi^{2}(\ldots)=0
$$

or

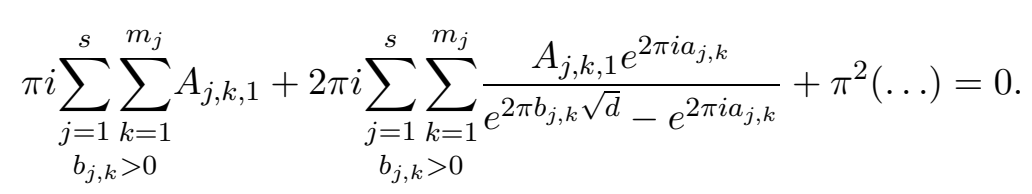

Now multiplying both sides of the last equality by

$$
\left.\prod_{\substack{j=1 \\ b_{j, k}>0}}^{s} \prod_{\substack{k=1 \\ m_{j}}}^{2 \pi b_{j, k} \sqrt{d}}-e^{2 \pi i a_{j, k}}\right)^{l_{j, k}}
$$

we get a contradiction with the algebraic independence of $\pi$ and $e^{\pi \sqrt{d}}$.

If (ii) is valid and $S$ is algebraic, then (16) can be rewritten as

$$
\pi C_{1}+2 \pi i \sum_{\substack{j=1 \\ b_{j, k}>0}}^{s} \sum_{\substack{k=1 \\ m_{j}}} \frac{A_{j, k, 1} e^{2 \pi i a_{j, k}}}{e^{2 \pi b_{j, k} \sqrt{d}}-e^{2 \pi i a_{j, k}}}+\pi^{2}(\ldots)=0 .
$$

If $C_{1} \neq 0$, then this is impossible by the same argument as above. If $C_{1}=0$, then multiplying both sides of (18) by (17) we get

$$
2 \pi i A_{j_{0}, k_{0}, 1} e^{2 \pi i a_{j_{0}, k_{0}}} e^{2 \pi\left(\beta-b_{j_{0}, k_{0}}\right) \sqrt{d}}+\cdots=0,
$$

which is impossible, and therefore $S$ is transcendental.

If condition (iii) holds, then the summands with the maximal power of $\pi$ in (16) have the form

$$
\pi^{l_{j_{0}, k_{0}}}\left( \pm \frac{A_{j_{0}, k_{0}, l_{j_{0}, k_{0}}}}{\left(l_{j_{0}, k_{0}}-1\right) !} p_{l_{j_{0}, k_{0}}-1}\left(-\cot \pi \alpha_{j_{0}, k_{0}}\right)+C_{j_{0}, k_{0}, l_{j_{0}, k_{0}}}\right),
$$

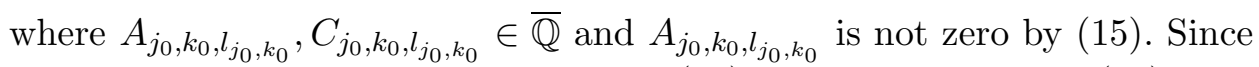
$\cot \pi \alpha_{j_{0}, k_{0}}$ is transcendental, the term (19) does not vanish in (16), and hence $S$ is transcendental. This completes the proof of the theorem.

REMARK 1.1. If under the assumptions of Theorem 1 we have $r_{1}=\cdots=$ $r_{s}=-1$, then $S$ is either zero or transcendental.

Corollary 4. If $a, b \in \mathbb{Z}, 4 b>a^{2}, m \in \mathbb{N}$, then the sum

$$
\sum_{n=0}^{\infty} \frac{P(n)}{\left(n^{2}+a n+b\right)^{m}}
$$


is transcendental for any polynomial $P(x) \in \overline{\mathbb{Q}}[x]$ such that

$$
\operatorname{deg} P(x) \leq 2 m-2 \quad \text { and } \quad P(-x)=P(x-a) .
$$

In particular, the sum of the series

$$
\sum_{n=0}^{\infty} \frac{\left(n^{2}+a n+c\right)^{k}}{\left(n^{2}+a n+b\right)^{m}}
$$

is transcendental for any $c, k \in \mathbb{Z}, 0 \leq k<m$.

Theorem 2. Let $P_{1}, \ldots, P_{s}, Q_{1}, \ldots, Q_{s} \in \overline{\mathbb{Q}}[x], m_{1}, \ldots, m_{s} \in \mathbb{N}, r_{1}, \ldots, r_{s}$ $\in \mathbb{Z}$ satisfy the following conditions: for any $1 \leq j \leq s, \operatorname{deg} P_{j} \leq \operatorname{deg} Q_{j}-1$,

$$
\frac{P_{j}(-x)}{Q_{j}(-x)}=(-1)^{r_{j}} \frac{P_{j}\left(r_{j}+x\right)}{Q_{j}\left(r_{j}+x\right)},
$$

$Q_{j}(x)=\prod_{k=1}^{2 m_{j}}\left(x-\alpha_{j, k}\right)^{l_{j, k}}$, where $\alpha_{j, k}=a_{j, k}+i b_{j, k} \sqrt{d} \in \mathbb{Q}(i \sqrt{d}) \backslash \mathbb{N}_{0}$, $k=1, \ldots, 2 m_{j}$, are distinct and such that $\alpha_{j, m_{j}+k}=r_{j}-\alpha_{j, k}, b_{j, k} \geq 0$, $l_{j, m_{j}+k}=l_{j, k} \in \mathbb{N}, k=1, \ldots, m_{j}$. Then the sum

$$
T=\sum_{n=0}^{\infty}\left(\frac{P_{1}(n)}{Q_{1}(n)}+\cdots+\frac{P_{s}(n)}{Q_{s}(n)}\right)(-1)^{n}
$$

is either a computable algebraic number or transcendental. Moreover, $T$ is transcendental if at least one of the following conditions holds:

(i) $b_{j_{0}, k_{0}}:=\min \left\{b_{j, k}: b_{j, k}>0\right\}$ is a unique minimum of the positive numbers $b_{j, k}$ and $\operatorname{res}_{z=\alpha_{j_{0}, k_{0}}} P_{j_{0}}(z) / Q_{j_{0}}(z) \neq 0$,

(ii) there exists a unique maximum $l_{j_{0}, k_{0}}$ of the sequence $l_{j, k}, 1 \leq j \leq s$, $1 \leq k \leq m_{j}$, and $b_{j_{0}, k_{0}}>0, P_{j_{0}}\left(\alpha_{j_{0}, k_{0}}\right) \neq 0$.

Proof. From Lemma 2 it follows that

$$
T=\sum_{j=1}^{s} \sum_{n=0}^{\infty} \frac{P_{j}(n)}{Q_{j}(n)}(-1)^{n}=\sum_{j=1}^{s} \sum_{k=1}^{2 m_{j}} \sum_{l=1}^{l_{j, k}} \frac{(-1)^{l-1}}{(l-1) !} A_{j, k, l} g^{(l-1)}\left(-\alpha_{j, k}\right),
$$

where the coefficients $A_{j, k, l}$ are defined in (15). According to (15) and (20) for $1 \leq k \leq m_{j}$ we have $A_{j, m_{j}+k, l}=(-1)^{r_{j}+l} A_{j, k, l}$. Then

$$
T=\sum_{j=1}^{s} \sum_{k=1}^{m_{j}} \sum_{l=1}^{l_{j, k}} \frac{(-1)^{l-1}}{(l-1) !} A_{j, k, l}\left(g^{(l-1)}\left(-\alpha_{j, k}\right)+(-1)^{r_{j}+l} g^{(l-1)}\left(\alpha_{j, k}-r_{j}\right)\right) \text {. }
$$

Now if for some pair $(j, k)$ we have $-\alpha_{j, k}$ and $\alpha_{j, k}-r_{j} \in \mathbb{N}$, then by $(5)$, (7), we get 


$$
\begin{aligned}
T= & C_{0}+\sum_{\substack{j=1 \\
\alpha_{j, k} \in \mathbb{Z}}}^{s} \sum_{\substack{k=1 \\
m_{j}}}^{m_{\text {even }}} \sum_{j, k}^{l_{j, k}} C_{j, l} \pi^{l} \\
& +\sum_{\substack{j=1 \\
\alpha_{j, k} \notin \mathbb{Z}}}^{m_{j}} \sum_{\substack{k=1 \\
l_{j, k}}}^{A_{j, k, l}}\left((-1)^{l-1} g^{(l-1)}\left(-\alpha_{j, k}\right)+g^{(l-1)}\left(\alpha_{j, k}+1\right)\right),
\end{aligned}
$$

where $C_{0}, C_{j, k, l} \in \overline{\mathbb{Q}}$. Hence, by (6) and Lemma 3, we have

$$
\begin{aligned}
& T=C_{0}+\sum_{j=1}^{s} \sum_{k=1}^{m_{j}} \sum_{l=1}^{L} C_{j, k, l} \pi^{l}-\sum_{j=1}^{s} \sum_{k=1}^{m_{j}} \sum_{l=1}^{l_{j, k}} \frac{A_{j, k, l}}{(l-1) !} \pi^{l} \frac{q_{l-1}\left(\cos \pi \alpha_{j, k}\right)}{\sin ^{l} \pi \alpha_{j, k}} \\
& \alpha_{j, k} \in \mathbb{Z} \quad l \text { even } \quad \alpha_{j, k} \notin \mathbb{Z}
\end{aligned}
$$

and according to Euler's formulas for cos and sin we conclude that either $T=C_{0}$ or $T$ is transcendental.

If $T$ is algebraic and condition (i) holds, then we rewrite (21) as

$$
\pi C_{1}+\pi \sum_{\substack{j=1 \\ b_{j, k}>0}}^{s} \sum_{\substack{k=1 \\ \text { m }}}^{m_{j}} \frac{A_{j, k, 1}}{\sin \pi \alpha_{j, k}}+\pi^{2}(\ldots)=0
$$

from which by the same argument as in the proof of Theorem 1(ii) and formula

$$
\frac{1}{\sin \pi \alpha_{j, k}}=-\frac{2 i e^{i \pi a_{j, k}} e^{\pi b_{j, k} \sqrt{d}}}{e^{2 \pi b_{j, k} \sqrt{d}}-e^{2 \pi i a_{j, k}}}
$$

we get a contradiction.

If condition (ii) is valid and $T$ is algebraic, then from (21) we have

$$
\pi^{l_{j_{0}, k_{0}}}\left(C_{j_{0}, k_{0}, l_{j_{0}, k_{0}}}-\frac{A_{j_{0}, k_{0}, l_{j_{0}, k_{0}}}}{\left(l_{j_{0}, k_{0}}-1\right) !} \frac{q_{j_{0}, k_{0}-1}\left(\cos \pi \alpha_{j_{0}, k_{0}}\right)}{\sin ^{l_{j_{0}}, k_{0}} \pi \alpha_{j_{0}, k_{0}}}\right)+\cdots=0,
$$

where $A_{j_{0}, k_{0}, l_{j_{0}, k_{0}}} \neq 0$ by (15). Now applying Lemma 3 we easily see that the term containing $\pi$ to the maximal power does not vanish and we get a contradiction with the algebraic independence of $\pi$ and $e^{\pi \sqrt{d}}$. This completes the proof.

REMARK 2.1. If under the assumptions of Theorem 2 we have $r_{1}=\cdots=$ $r_{s}=-1$, then $T$ is either zero or transcendental.

REMARK 2.2. We note that there are alternative proofs of formulas (16), (21) based on application of the residue theorem to the complex integrals

$$
\frac{1}{2 \pi i} \int_{L_{N}}\left(\sum_{j=1}^{s} \frac{P_{j}(z)}{Q_{j}(z)}\right)(\pi \cot \pi z) d z \quad \text { and } \quad \frac{1}{2 \pi i} \int_{L_{N}}\left(\sum_{j=1}^{s} \frac{P_{j}(z)}{Q_{j}(z)}\right) \frac{\pi}{\sin \pi z} d z,
$$


where $L_{N}$ is a square contour with vertices $(N+1 / 2)( \pm 1 \pm i)$. (See also [3, Theorem 2].)

Corollary 5. Let $a, b \in \mathbb{Z}, 4 b>a^{2}$, and $m \in \mathbb{N}$. Then for any polynomial $P(x) \in \overline{\mathbb{Q}}[x]$ such that $\operatorname{deg} P(x)<2 m, P(-x)=(-1)^{a} P(x-a)$, the sum

$$
\sum_{n=0}^{\infty} \frac{(-1)^{n} P(n)}{\left(n^{2}+a n+b\right)^{m}}
$$

is transcendental. In particular, if $k \in \mathbb{Z}, 0 \leq k<2 m$, and the numbers $k$, $a$ have the same parity, then the sum

$$
\sum_{n=0}^{\infty} \frac{(-1)^{n}(n+a / 2)^{k}}{\left(n^{2}+a n+b\right)^{m}}
$$

is transcendental.

TheOREM 3. Let $f: \mathbb{Z} \rightarrow \overline{\mathbb{Q}}$ be periodic with period $q \in \mathbb{N}$. Suppose that $r \in \mathbb{Z}, m, l_{1}, \ldots, l_{m} \in \mathbb{N}, P(x), Q(x) \in \overline{\mathbb{Q}}[x]$,

$$
\frac{P(-x)}{Q(-x)}= \pm \frac{P(x+q r)}{Q(x+q r)},
$$

$Q(x)=\left(x-\alpha_{0}\right) \prod_{k=1}^{2 m}\left(x-\alpha_{k}\right)^{l_{k}}$, where $\alpha_{0}=q r / 2, \alpha_{k}=a_{k}+i b_{k} \sqrt{d} \in$ $\mathbb{Q}(i \sqrt{d}) \backslash \mathbb{N}, k=1, \ldots, 2 m$, are distinct, $\alpha_{m+k}=q r-\alpha_{k}, l_{m+k}=l_{k}, b_{k} \geq 0$, $k=1, \ldots, m$, and $f$ is an even or odd function according to whether we have the "plus" or "minus" sign in (22). Suppose further that the series

$$
U=\sum_{n=1}^{\infty} \frac{P(n)}{Q(n)} f(n)
$$

converges. Then $U$ is either a computable algebraic number or transcendental. Moreover, $U$ is transcendental if at least one of the following conditions holds:

(i) $P(q r / 2)=0$ and

$$
\sum_{\substack{t=1 \\ t-\alpha_{k} \notin q \mathbb{Z}}}^{q} \sum_{\substack{k=1 \\ t=\alpha_{k}}}^{m} f(t) \underset{Q(z)}{\operatorname{res}} \frac{P(z)}{Q(z)}
$$

(ii) $P(q r / 2)=0, b_{k_{0}}:=\min \left\{b_{k}>0\right\}$ is a unique minimum of the positive numbers $b_{k}, \operatorname{res}_{z=\alpha_{k_{0}}} P(z) / Q(z) \neq 0$ and $\sum_{t=1}^{q} f(t) e^{-2 \pi i t / q} \neq 0$,

(iii) $\sum_{\substack{t=1 \\ t-\alpha_{k} \notin q \mathbb{Z}}}^{q-1} \sum_{\substack{k=1 \\ z=\alpha_{k}}}^{m} f(t) \underset{Q(z)}{\operatorname{res}} \frac{P(z)}{2} \frac{i}{Q^{\prime}(q r / 2)} \sum_{\substack{t=1 \\ t \neq q / 2}}^{q-1} f(t) \cot \left(\frac{\pi t}{q}+\pi\left\{\frac{r}{2}\right\}\right)$ and $P(q r / 2) \neq 0$, where $\{x\}$ denotes the fractional part of $x$. 
Proof. By Lemma 1, using the partial fraction expansion

$$
\frac{P(x)}{Q(x)}=\sum_{k=1}^{2 m} \sum_{l=1}^{l_{k}} \frac{A_{k, l}}{\left(x-\alpha_{k}\right)^{l}}+\frac{A_{0,1}}{x-q r / 2},
$$

where the coefficients $A_{k, l}$ are defined in (11) with $\alpha_{k}$ replaced by $-\alpha_{k}$ and $A_{0,1}=P(q r / 2) / Q^{\prime}(q r / 2)$, we have

$$
U=\sum_{t=1}^{q} f(t) \sum_{k=1}^{2 m} \sum_{l=1}^{l_{k}} \frac{(-1)^{l}}{(l-1) !} \frac{A_{k, l}}{q^{l}} \psi^{(l-1)}\left(\frac{t-\alpha_{k}}{q}\right)-\frac{A_{0,1}}{q} \sum_{t=1}^{q} f(t) \psi\left(\frac{t}{q}-\frac{r}{2}\right) .
$$

By (22), for $1 \leq k \leq m, 1 \leq l \leq l_{k}$, it easily follows that $A_{m+k, l}=$ $\pm(-1)^{l} A_{k, l}$.

To prove the theorem, we first assume that $P(q r / 2)=0$. Then taking into account that $f(t)= \pm f(-t)$ and $f$ is a $q$-periodic function we have

$$
\begin{aligned}
U= & \sum_{t=1}^{q} f(t) \sum_{k=1}^{m} \sum_{l=1}^{l_{k}} \frac{(-1)^{l}}{(l-1) !} \frac{A_{k, l}}{q^{l}}\left(\psi^{(l-1)}\left(\frac{t-\alpha_{k}}{q}\right)\right. \\
& \left. \pm(-1)^{l} \psi^{(l-1)}\left(\frac{t-\alpha_{m+k}}{q}\right)\right) \\
= & \sum_{t=1}^{q} \sum_{k=1}^{m} \sum_{l=1}^{l_{k}} \frac{(-1)^{l} f(t)}{(l-1) !} \frac{A_{k, l}}{q^{l}} \psi^{(l-1)}\left(\frac{t-\alpha_{k}}{q}\right) \\
& +\sum_{t=1}^{q} \sum_{k=1}^{m} \sum_{l=1}^{l_{k}} \frac{A_{k, l} f(q-t)}{(l-1) ! q^{l}} \psi^{(l-1)}\left(\frac{t-\alpha_{m+k}}{q}\right) \\
= & A+\sum_{t=1}^{q} f(t) \sum_{k=1}^{m} \sum_{l=1}^{l_{k}} \frac{(-1)^{l}}{(l-1) !} \\
& \times \frac{A_{k, l}}{q^{l}}\left(\psi^{(l-1)}\left(\frac{t-\alpha_{k}}{q}\right)+(-1)^{l} \psi^{(l-1)}\left(1-r-\frac{t-\alpha_{k}}{q}\right)\right),
\end{aligned}
$$

where $A=-f(q) \sum_{k=1}^{m} \sum_{l=1}^{l_{k}} A_{k, l} / \alpha_{m+k}^{l} \in \overline{\mathbb{Q}}$. Now by (3), (7) and Lemma 3 we get

$$
\begin{aligned}
U= & C_{0}+\sum_{\substack{t=1 \\
t-\alpha_{k} \in q \mathbb{Z}}}^{q} \sum_{\substack{k=1 \\
l=2}}^{m} \sum_{t, k, l}^{l_{k}} C^{l} \\
& -\sum_{\substack{t=1 \\
t-\alpha_{k} \notin q \mathbb{Z}}}^{q} \sum_{\substack{k=1 \\
l=1}}^{l_{k}} \frac{(-\pi)^{l} f(t) A_{k, l}}{q^{l}} p_{l-1}\left(\cot \left(\frac{\pi\left(t-\alpha_{k}\right)}{q}\right)\right)
\end{aligned}
$$

with $C_{0}, C_{t, k, l} \in \overline{\mathbb{Q}}$, from which it follows that $U$ is either equal to $C_{0} \in \overline{\mathbb{Q}}$ 
or transcendental. If condition (i) or (ii) holds, then arguing as in the proof of Theorem 1(i), (ii) we find that $U$ is transcendental.

If $P(q r / 2) \neq 0$, then $P(-x)=P(x+q r)$ and thus $f$ is an odd function by the hypothesis. Arguing as above we deduce that $A_{k+m, l}=(-1)^{l-1} A_{k, l}$, $1 \leq k \leq m, 1 \leq l \leq l_{k}$, and

$$
\begin{aligned}
U= & \sum_{t=1}^{q-1} f(t) \sum_{k=1}^{m} \sum_{l=1}^{l_{k}} \frac{(-1)^{l}}{(l-1) !} \\
& \times \frac{A_{k, l}}{q^{l}}\left(\psi^{(l-1)}\left(\frac{t-\alpha_{k}}{q}\right)+(-1)^{l} \psi^{(l-1)}\left(1-r-\frac{t-\alpha_{k}}{q}\right)\right) \\
& -\frac{A_{0,1}}{2 q} \sum_{t=1}^{q-1} f(t)\left(\psi\left(\frac{t}{q}-\frac{r}{2}\right)-\psi\left(1-\frac{t}{q}-\frac{r}{2}\right)\right) .
\end{aligned}
$$

As is easily seen, if $q$ is even, then $f(q / 2)=0$ and we may assume that $t \neq q / 2$ in the last sum. Now by (2), for a positive integer $t \leq q-1, t \neq q / 2$, we have

$$
\begin{aligned}
\psi\left(\frac{t}{q}-\frac{r}{2}\right) & =C+\psi\left(\frac{t}{q}-\frac{r}{2}+\left[\frac{r+1}{2}\right]\right), \\
\psi\left(1-\frac{t}{q}-\frac{r}{2}\right) & =\widetilde{C}+\psi\left(1-\frac{t}{q}-\frac{r}{2}+\left[\frac{r}{2}\right]\right),
\end{aligned}
$$

where $C, \widetilde{C} \in \overline{\mathbb{Q}}$ and $[x]$ denotes the integer part of $x$. Now by (3), (23) and Lemma 3 we get

$$
\begin{aligned}
U= & C_{1}+\sum_{\substack{t=1 \\
t-\alpha_{k} \in q \mathbb{Z}}}^{q-1} \sum_{\substack{k=1 \\
l=2}}^{m} \sum_{t, k, l} \pi^{l} \\
& -\sum_{\substack{t=1 \\
t-1}}^{m} \sum_{\substack{k=1 \\
t-\alpha_{k} \notin q \mathbb{Z}}}^{l_{k}} \frac{A_{k, l} \pi^{l} f(t)}{(-q)^{l}} p_{l-1}\left(\cot \left(\frac{\pi\left(t-\alpha_{k}\right)}{q}\right)\right) \\
& +\frac{A_{0,1} \pi}{2 q} \sum_{t=1}^{q-1} f(t) \cot \left(\frac{\pi t}{q}+\pi\left\{\frac{r}{2}\right\}\right)
\end{aligned}
$$

with $C_{1}, C_{t, k, l} \in \overline{\mathbb{Q}}$, and therefore $U$ is either equal to $C_{1}$ or transcendental. If $r=0$, i.e., if $P(x)$ and $Q(x)$ are even and odd polynomials respectively, then $C_{1}=0$ and hence $U$ is either zero or transcendental. If condition (iii) is valid, then the coefficient of $\pi$ does not vanish in (24) and we conclude that $U$ is transcendental. This completes the proof of the theorem.

REMARK 3.1. If under the assumptions of Theorem 3 we have $r=0$, then either $U=-f(q) \sum_{k=1}^{m} \sum_{l=1}^{l_{k}} A_{k, l} / \alpha_{k+m}^{l}$ or $U$ is transcendental. 
TheOREM 4. Let $k \in \mathbb{N}, r \in \mathbb{Z}, q r / 2 \notin \mathbb{N}, P(x) \in \overline{\mathbb{Q}}[x]$ and $P(-x)=$ $\pm P(x+q r)$. Let $f: \mathbb{Z} \rightarrow \overline{\mathbb{Q}}$ be an even or odd periodic function with period $q \in \mathbb{N}$ depending on whether $k$ and $\operatorname{deg} P(x)$ have the same parity or not. Suppose further that the series

$$
U=\sum_{n=1}^{\infty} \frac{f(n) P(n)}{(n-q r / 2)^{k}}
$$

converges. Then the sum $U$ is either a computable algebraic number or transcendental. In particular, if $r=0$, then $U$ is either zero or transcendental.

Proof. For the rational function $P(x) /(x-q r / 2)^{k}$ we have the following partial fraction expansion:

$\frac{P(x)}{(x-q r / 2)^{k}}=\sum_{l=0}^{[(\operatorname{deg} P) / 2]} \frac{A_{l}}{(x-q r / 2)^{k-\delta-2 l}} \quad$ with $\quad A_{l}=\frac{1}{(2 l+\delta) !} P^{(2 l+\delta)}\left(\frac{q r}{2}\right)$

and $\delta$ equal to 0 or 1 according to whether $P(-x)=P(x+q r)$ or $P(-x)=$ $-P(x+q r)$. Then by Lemma 1 , we get

$$
U=\sum_{t=1}^{q} f(t) \sum_{l=0}^{[(\operatorname{deg} P) / 2]} \frac{(-1)^{k-\delta-1}}{(k-\delta-2 l-1) !} \frac{A_{l}}{q^{k-\delta-2 l}} \psi^{(k-\delta-2 l-1)}\left(\frac{t}{q}-\frac{r}{2}\right) .
$$

Note that if $k$ and $\operatorname{deg} P$ have the same (distinct) parity, then $k-\delta$ is even (odd) and $f$ is an even (odd) function by the hypothesis. Thus we have $f(t)=(-1)^{k-\delta} f(q-t)$ and

$$
2 U=\sum_{t=1}^{q} \sum_{l=0}^{[(\operatorname{deg} P) / 2]} \frac{(-1)^{k-\delta-1} f(t)-f(q-t)}{(k-\delta-2 l-1) !} \frac{A_{l}}{q^{k-\delta-2 l}} \psi^{(k-\delta-2 l-1)}\left(\frac{t}{q}-\frac{r}{2}\right)
$$

or

$$
\begin{aligned}
2 U= & \sum_{t=1}^{q-1} f(t) \sum_{l=0}^{[(\operatorname{deg} P) / 2]} \frac{(-1)^{k-\delta-1}}{(k-\delta-2 l-1) !} \frac{A_{l}}{q^{k-\delta-2 l}}\left(\psi^{(k-\delta-2 l-1)}\left(\frac{t}{q}-\frac{r}{2}\right)\right. \\
& \left.+(-1)^{k-\delta} \psi^{(k-\delta-2 l-1)}\left(1-\frac{t}{q}-\frac{r}{2}\right)\right)+\widetilde{U}
\end{aligned}
$$

where

$$
\begin{aligned}
\widetilde{U}= & \left(f(q)+(-1)^{k-\delta} f(0)\right) \\
& \times \sum_{l=0}^{[(\operatorname{deg} P) / 2]} \frac{(-1)^{k-\delta-1}}{(k-\delta-2 l-1) !} \frac{A_{l}}{q^{k-\delta-2 l}} \psi^{(k-\delta-2 l-1)}\left(1-\frac{r}{2}\right) .
\end{aligned}
$$

It can be easily seen that $\widetilde{U}=0$ if $f$ is an odd function; if $f$ is even, then 
$k-\delta$ is even and by (7) we have

$$
\widetilde{U}=C+\sum_{l=0}^{[(\operatorname{deg} P) / 2]} C_{l} \pi^{k-\delta-2 l}
$$

with algebraic coefficients $C, C_{l}$. From (23), (3), (7) and Lemma 3 it follows that

$$
\begin{aligned}
\psi^{(k-\delta-2 l-1)}\left(\frac{t}{q}-\frac{r}{2}\right)+(-1)^{k-\delta} \psi^{(k-\delta-2 l-1)}\left(1-\frac{t}{q}-\frac{r}{2}\right) & \in \mathbb{Q} \pi^{k-\delta-2 l}+\mathbb{Q} .
\end{aligned}
$$

Finally, by (25)-(28), we find

$$
U=\widetilde{C}+\sum_{l=0}^{[(\operatorname{deg} P) / 2]} \widetilde{C}_{l} \pi^{k-\delta-2 l}
$$

with $\widetilde{C}, \widetilde{C}_{l} \in \overline{\mathbb{Q}}$, and therefore either $U$ is equal to $\widetilde{C}$ or $U \notin \overline{\mathbb{Q}}$. If $r=0$, then from (25), (26) it easily follows that $U$ is either zero or transcendental. This completes the proof of the theorem.

The special case of Theorem 4 for the number $U=L(k, \chi)=\sum_{n=1}^{\infty} \chi(n) / n^{k}$, where $\chi$ is an even (or odd) Dirichlet character, was proved in $[10, \S 6]$.

Now consider several applications of Theorem 3 which gives us means to construct new examples of transcendental numbers. If in Theorem 3 we put $f(n)=\chi(n)$, where $\chi(n)$ is a Dirichlet character $\bmod q$, then the Gauss sum

$$
\tau(\chi)=\sum_{k=1}^{q} \chi(k) e^{-2 \pi i k / q}
$$

is never zero when $\chi$ is a primitive character (see [4, Ch. 8]). Namely, we have $|\tau(\chi)|=\sqrt{q}$. This gives us the following.

COROllary 6. Let $q>1$ be an integer and $\chi$ be a primitive character mod $q$. Suppose that $P(x) \in \overline{\mathbb{Q}}[x], P(-x)= \pm P(x+q r), Q(x)=$ $\prod_{k=1}^{2 m}\left(x-\alpha_{k}\right)^{l_{k}}$ for some $m, l_{1}, \ldots, l_{2 m} \in \mathbb{N}, r \in \mathbb{Z}$, where $\alpha_{k}=a_{k}+i b_{k} \sqrt{d} \in$ $\mathbb{Q}(i \sqrt{d}) \backslash \mathbb{N}, k=1, \ldots, 2 m$, are distinct numbers such that $\alpha_{m+k}=q r-\alpha_{k}$, $b_{k} \geq 0, l_{m+k}=l_{k}, k=1, \ldots, m$, and $\chi$ is an even (resp. odd) character if $\operatorname{deg} P$ is even (resp. odd). If $b_{k_{0}}:=\min \left\{b_{k}>0\right\}$ is a unique minimum of the positive numbers $b_{k}$ and $\operatorname{res}_{z=\alpha_{k_{0}}} P(z) / Q(z) \neq 0$, then the sum

$$
\sum_{n=1}^{\infty} \frac{P(n)}{Q(n)} \chi(n)
$$

is transcendental. 
COROLlary 7 . Let $q>1$ be a square-free integer with $q \equiv 1(\bmod 4)$, and let $\left(\frac{n}{q}\right)$ denote Jacobi's symbol. Then

$$
\sum_{n=1}^{\infty} \frac{P(n)}{Q(n)}\left(\frac{n}{q}\right) \notin \overline{\mathbb{Q}}
$$

where $P(x) \in \overline{\mathbb{Q}}[x], P(-x)=P(x+q r)$ and $Q(x)$ is as in Corollary 6. In particular, the sum

$$
\sum_{n=1}^{\infty} \frac{\left(\frac{n}{q}\right)}{\left(n^{2}+q r n+b\right)^{m}}
$$

is transcendental for any $m \in \mathbb{N}, b, r \in \mathbb{Z}$ such that $q^{2} r^{2}<4 b$.

COROLlary 8. Let $q>1$ be a square-free integer with $q \equiv 3(\bmod 4)$. Then

$$
\sum_{n=1}^{\infty} \frac{P(n)}{Q(n)}\left(\frac{n}{q}\right) \notin \overline{\mathbb{Q}}
$$

where $P(x) \in \overline{\mathbb{Q}}[x], P(-x)=-P(x+q r)$ and $Q(x)$ is as in Corollary 6. In particular, the sum

$$
\sum_{n=1}^{\infty}\left(\frac{n}{q}\right) \frac{(n+q r / 2)^{2 m-1}}{\left(n^{2}+q r n+b\right)^{m}}
$$

is transcendental for any $m \in \mathbb{N}, b, r \in \mathbb{Z}$ such that $q^{2} r^{2}<4 b$.

If $\chi_{0}$ is the principal character $\bmod q$, then

$$
\sum_{n=1}^{q} \chi_{0}(n)=\varphi(q), \quad \tau\left(\chi_{0}\right)=\sum_{\substack{k=1 \\(k, q)=1}}^{q} e^{-2 \pi i k / q}=\mu(q),
$$

where $\varphi$ and $\mu$ are the Euler and Möbius functions, respectively (see [11, Ch. 3]) and we have

COROLlary 9. If $q>1$ is a square-free integer and $\chi_{0}$ is the principal character mod $q$, then the sum

$$
\sum_{n=1}^{\infty} \frac{P(n)}{Q(n)} \chi_{0}(n)
$$

is transcendental, where $P(x) \in \overline{\mathbb{Q}}[x], P(-x)=P(x+q r)$ and the polynomial $Q(x)$ is as in Corollary 6. In particular, the sum of the series

$$
\sum_{n=1}^{\infty} \frac{\chi_{0}(n)}{\left(n^{2}+q r n+b\right)^{m}}
$$

is transcendental for any $m \in \mathbb{N}, b, r \in \mathbb{Z}$ such that $q^{2} r^{2}<4 b$. 
COROllary 10. Let $q>1$ be an integer and $\chi_{0}$ the principal character mod q. Suppose that $P(x), Q(x) \in \overline{\mathbb{Q}}[x], P(-x)=P(x+q r)$ and $Q(x)=$ $\prod_{k=1}^{2 m}\left(x-\alpha_{k}\right)^{l_{k}}$ for some $m, l_{1}, \ldots, l_{2 m} \in \mathbb{N}, r \in \mathbb{Z}$, where $\alpha_{k}=a_{k}+i b_{k} \sqrt{d} \in$ $\mathbb{Q}(i \sqrt{d}) \backslash \mathbb{Q}, k=1, \ldots, 2 m$, are distinct and such that $\alpha_{k+m}=\alpha_{k}, b_{k} \geq 0$, $l_{k+m}=l_{k}, k=1, \ldots, m$. If $\sum_{k=1}^{m} \operatorname{res}_{z=\alpha_{k}} P(z) / Q(z) \neq 0$, then the sum

$$
\sum_{n=1}^{\infty} \frac{P(n)}{Q(n)} \chi_{0}(n)
$$

is transcendental.

Corollary 11. Let $f: \mathbb{Z} \rightarrow \overline{\mathbb{Q}}$ be odd, periodic with period $q \in \mathbb{N}$. Then the sum

$$
\sum_{n=1}^{\infty} \frac{P(n) f(n)}{n\left(n^{2}+b\right)^{m}}
$$

is either zero or transcendental for any $m, b \in \mathbb{N}$ and any even polynomial $P(x)$ with $\operatorname{deg} P \leq 2 m$.

\section{Transcendence of trigonometric series}

TheOREm 5. Suppose that $\beta_{1}, \ldots, \beta_{s} \in[0,2)$ are distinct rational numbers, $Q(x), P_{1}(x), \ldots, P_{s}(x) \in \overline{\mathbb{Q}}[x], Q(x)=\left(x-\alpha_{1}\right)^{l_{1}} \ldots\left(x-\alpha_{m}\right)^{l_{m}}$, where $\alpha_{1}, \ldots, \alpha_{m} \in \mathbb{Q}(i \sqrt{d}) \backslash \mathbb{Z}$ are distinct, $l_{1}, \ldots, l_{m} \in \mathbb{N}, h(n)=\sum_{j=1}^{s} P_{j}(n) e^{i \pi \beta_{j} n}$, and for $1 \leq j \leq s$,

$$
\operatorname{deg} P_{j}(x) \leq \begin{cases}\operatorname{deg} Q(x)-1 & \text { if } \beta_{j}>0, \\ \operatorname{deg} Q(x)-2 & \text { if } \beta_{j}=0 .\end{cases}
$$

Then the sum

$$
V=\sum_{n=-\infty}^{\infty} \frac{h(n)}{Q(n)}
$$

is either zero or transcendental.

Proof. We consider the complex integral

$$
I_{N}=\frac{1}{2 \pi i} \int_{L_{N}} \frac{h^{-}(z)}{Q(z)} \frac{\pi}{\sin \pi z} d z,
$$

where $h^{-}(z)=\sum_{j=1}^{s} P_{j}(z) e^{i \pi\left(\beta_{j}-1\right) z}, L_{N}$ is a square contour with vertices $(N+1 / 2)( \pm 1 \pm i)$, and $N$ is a large positive integer such that $\alpha_{1}, \ldots, \alpha_{m}$ are inside $L_{N}$. For $z= \pm(N+1 / 2)+i y, y \in[-N-1 / 2, N+1 / 2]$, we have

$$
\left|\frac{1}{\sin \pi z}\right|=\frac{2}{e^{\pi y}+e^{-\pi y}}
$$


and therefore,

$$
\begin{aligned}
\left|\frac{P_{j}(z) e^{i \pi\left(\beta_{j}-1\right) z}}{Q(z) \sin \pi z}\right| & =\frac{2\left|P_{j}(z)\right|}{\left|Q_{j}(z)\right|\left(e^{\pi \beta_{j} y}+e^{\pi\left(\beta_{j}-2\right) y}\right)} \\
& \leq 2 \frac{\left|P_{j}(z)\right|}{\left|Q_{j}(z)\right|} e^{-\pi|y| \min \left\{\beta_{j}, 2-\beta_{j}\right\}} .
\end{aligned}
$$

If $\beta_{j}=0$, then from (29) it follows that

$$
\begin{aligned}
\left|\frac{1}{2 \pi i} \int_{\substack{z= \pm(N+1 / 2)+i y \\
-N-1 / 2 \leq y \leq N+1 / 2}} \frac{P_{j}(z) e^{i \pi\left(\beta_{j}-1\right) z}}{Q(z)} \frac{\pi}{\sin \pi z} d z\right| \\
\leq \int_{-N-1 / 2}^{N+1 / 2} \frac{\left|P_{j}(z)\right|}{\left|Q_{j}(z)\right|} d y=O\left(\frac{1}{N}\right) .
\end{aligned}
$$

If $0<\beta_{j}<2$, then (29) implies

$$
\begin{gathered}
\left|\frac{1}{2 \pi i} \int_{\substack{z= \pm(N+1 / 2)+i y \\
-N-1 / 2 \leq y \leq N+1 / 2}} \frac{P_{j}(z) e^{i \pi\left(\beta_{j}-1\right) z}}{Q(z)} \frac{\pi}{\sin \pi z} d z\right| \\
\leq O\left(\frac{1}{N}\right) \int_{-N-1 / 2}^{N+1 / 2} e^{-\pi|y| \min \left\{\beta_{j}, 2-\beta_{j}\right\}} d y=O\left(\frac{1}{N}\right) .
\end{gathered}
$$

If $z=x \pm i(N+1 / 2), x \in[-N-1 / 2, N+1 / 2]$, then

$$
\left|\frac{1}{\sin \pi z}\right|=\frac{2}{e^{\pi(N+1 / 2)}-e^{-\pi(N+1 / 2)}}
$$

and

$$
\begin{aligned}
\left|\frac{P_{j}(z) e^{i \pi\left(\beta_{j}-1\right) z}}{Q(z) \sin \pi z}\right| & \leq \frac{2\left|P_{j}(z)\right|}{|Q(z)|} \frac{e^{\pi\left|\beta_{j}-1\right|(N+1 / 2)}}{e^{\pi(N+1 / 2)}-e^{-\pi(N+1 / 2)}} \\
& = \begin{cases}O\left(\frac{1}{N^{2}}\right) & \text { if } \beta_{j}=0 \\
O\left(\frac{1}{N e^{\pi\left(1-\left|\beta_{j}-1\right|\right) N}}\right) & \text { if } 0<\beta_{j}<2 .\end{cases}
\end{aligned}
$$

Therefore, by (30)-(32), we conclude that $I_{N}=O\left(N^{-1}\right)$ as $N \rightarrow \infty$. On the other hand, by the residue theorem we have

$$
I_{N}-\sum_{k=1}^{m} \operatorname{res}_{z=\alpha_{k}}\left(\frac{h^{-}(z)}{Q(z)} \frac{\pi}{\sin \pi z}\right)=\sum_{k=-N}^{N} \underset{z=k}{\operatorname{res}}\left(\frac{h^{-}(z)}{Q(z)} \frac{\pi}{\sin \pi z}\right)=\sum_{k=-N}^{N} \frac{h(k)}{Q(k)} .
$$


Now letting $N$ tend to infinity we get

$$
V=-\sum_{k=1}^{m} \operatorname{res}_{z=\alpha_{k}}\left(\frac{\pi h^{-}(z)}{Q(z) \sin \pi z}\right)=\left.\sum_{k=1}^{m} \frac{-\pi}{\left(l_{k}-1\right) !}\left(\frac{h^{-}(z)\left(z-\alpha_{k}\right)^{l_{k}}}{Q(z) \sin \pi z}\right)^{\left(l_{k}-1\right)}\right|_{z=\alpha_{k}},
$$

which implies that $V \in \overline{\mathbb{Q}}\left(\pi, e^{\pi \sqrt{d} / B}\right)$ for some $B \in \mathbb{N}$, and hence either $V=0$ or $V \notin \overline{\mathbb{Q}}$.

COROLlarY 12. If in addition to the assumptions of Theorem $5, Q(x)$ is an even polynomial, then the sum

$$
W=\sum_{n=0}^{\infty} \frac{h(n)+h(-n)}{Q(n)}
$$

is either $h(0) / Q(0)$ or transcendental.

Corollary 13. Suppose that $\beta_{1}, \beta_{2} \in(0,1) \cup(1,2)$ are rational numbers, $Q(x), P_{1}(x), P_{2}(x) \in \overline{\mathbb{Q}}[x]$ such that $P_{1}(x), Q(x)$ are even polynomials, $P_{2}(x)$ is an odd polynomial, $\operatorname{deg} P_{j}(x) \leq \operatorname{deg} Q(x)-1, j=1,2$, and all roots of $Q(x)$ belong to $\mathbb{Q}(i \sqrt{d}) \backslash \mathbb{Z}$. Then the trigonometric series

$$
W=\frac{P_{1}(0)}{2 Q(0)}+\sum_{n=1}^{\infty} \frac{P_{1}(n) \cos \left(\pi \beta_{1} n\right)+P_{2}(n) \sin \left(\pi \beta_{2} n\right)}{Q(n)}
$$

is either zero or transcendental.

Proof. We define

$$
h(n)= \begin{cases}\frac{1}{2} P_{1}(n) e^{i \pi \beta_{1} n}-\frac{1}{2} i P_{2}(n) e^{i \pi \beta_{2} n} & \text { if } \beta_{1} \neq \beta_{2}, \\ \frac{1}{2} P_{1}(n) e^{i \pi \beta_{1} n}+\frac{1}{2} i P_{2}(n) e^{i \pi\left(2-\beta_{1}\right) n} & \text { if } \beta_{1}=\beta_{2},\end{cases}
$$

and consider the sum

$\sum_{n=0}^{\infty} \frac{h(n)+h(-n)}{Q(n)}-\frac{h(0)}{Q(0)}=\frac{P_{1}(0)}{2 Q(0)}+\sum_{n=1}^{\infty} \frac{P_{1}(n) \cos \left(\pi \beta_{1} n\right)+P_{2}(n) \sin \left(\pi \beta_{2} n\right)}{Q(n)}$,

which, by Corollary 12, is either zero or transcendental.

5. Schanuel's conjecture and infinite sums. For more general set of roots of the polynomials $Q_{j}(x)$, when not all $\alpha_{j, k}$ are in $\mathbb{Q}(i \sqrt{d})$, we give some statements on the transcendence of the sums $S, T, U, V$ provided that the Schanuel conjecture holds (see [12, §3.1], [8, §10.7.G]).

Schanuel Conjecture (S). If $\alpha_{1}, \ldots, \alpha_{n} \in \mathbb{C}$ are linearly independent over $\mathbb{Q}$, then the transcendence degree over $\mathbb{Q}$ of the field $\mathbb{Q}\left(\alpha_{1}, \ldots, \alpha_{n}\right.$, $\left.e^{\alpha_{1}}, \ldots, e^{\alpha_{n}}\right)$ is at least $n$.

We formulate the following propositions, which are consequences of (S): 
Conjecture $\left(\mathrm{S}_{1}\right)$. Let $P_{1}, \ldots, P_{s}, Q_{1}, \ldots, Q_{s} \in \overline{\mathbb{Q}}[x], r_{1}, \ldots, r_{s} \in \mathbb{Z}$, where for any $1 \leq j \leq s$ the polynomials $P_{j}, Q_{j}$ satisfy the following conditions: $\operatorname{deg} P_{j} \leq \operatorname{deg} Q_{j}-2, Q_{j}\left(r_{j} / 2\right) \neq 0, Q_{j}(n) \neq 0, n=0,1, \ldots$, and

$$
\frac{P_{j}(-x)}{Q_{j}(-x)}=\frac{P_{j}\left(r_{j}+x\right)}{Q_{j}\left(r_{j}+x\right)} \text {. }
$$

Then the sum

$$
S=\sum_{n=0}^{\infty}\left(\frac{P_{1}(n)}{Q_{1}(n)}+\cdots+\frac{P_{s}(n)}{Q_{s}(n)}\right)
$$

is either a computable algebraic number or transcendental.

Proof. Under the conditions stated above, we see that for $1 \leq j \leq s$, $Q_{j}(x)=\prod_{k=1}^{2 m_{j}}\left(x-\alpha_{j, k}\right)^{l_{j, k}}$, where $\alpha_{j, k}$ are distinct algebraic numbers distinct from non-negative integers and such that $\alpha_{j, m_{j}+k}=r_{j}-\alpha_{j, k}, l_{j, m_{j}+k}=l_{j, k}$ $\in \mathbb{N}, k=1, \ldots, m_{j}$. Therefore, from (16) we have

$$
\begin{aligned}
& S=C_{0}+\sum_{\substack{j=1 \\
\alpha_{j, k} \in \mathbb{Z}}}^{s} \sum_{\substack{l=1 \\
l \text { even }}}^{m_{j}} \sum_{\substack{l, k \\
l_{j, k}}} C_{j, k} \pi^{l} \\
& +\sum_{j=1}^{s} \sum_{k=1}^{m_{j}} \sum_{l=1}^{l_{j, k}} \frac{(-1)^{l-1} A_{j, k, l}}{(l-1) !} \pi^{l} p_{l-1}\left(-\cot \pi \alpha_{j, k}\right), \\
& \alpha_{j, k} \notin \mathbb{Z}
\end{aligned}
$$

where $C_{0}$ and all the coefficients $C_{j, k, l}, A_{j, k, l}$ are algebraic numbers. From (33) it follows that $S$ is equal to $C_{0}$ or transcendental by (S). Indeed, suppose that $S \neq C_{0}$ and $S$ is algebraic. Assume that the numbers

$$
\frac{1}{\lambda}, \frac{\alpha_{j_{1}, k_{1}}}{\lambda_{1}}, \ldots, \frac{\alpha_{j_{l}, k_{l}}}{\lambda_{l}},
$$

where $\lambda_{1}, \ldots, \lambda_{l} \in \mathbb{N}$, are linearly independent over $\mathbb{Q}$ and all the other roots $\alpha_{j, k}$ are $\mathbb{Z}$-linear combinations of (34). Then the numbers

$$
\frac{\pi i}{\lambda}, \frac{\pi i \alpha_{j_{1}, k_{1}}}{\lambda_{1}}, \ldots, \frac{\pi i \alpha_{j_{l}, k_{l}}}{\lambda_{l}}
$$

are also linearly independent over $\mathbb{Q}$. Put

$$
\begin{aligned}
K & =\overline{\mathbb{Q}}\left(\frac{\pi i}{\lambda}, \frac{\pi i \alpha_{j_{1}, k_{1}}}{\lambda_{1}}, \ldots, \frac{\pi i \alpha_{j_{l}, k_{l}}}{\lambda_{l}}, e^{\pi i \alpha_{j_{1}, k_{1}} / \lambda_{1}}, \ldots, e^{\pi i \alpha_{j_{l}, k_{l}} / \lambda_{l}}\right) \\
& =\overline{\mathbb{Q}}\left(\frac{\pi i}{\lambda}, e^{\pi i \alpha_{j_{1}, k_{1}} / \lambda_{1}}, \ldots, e^{\pi i \alpha_{j_{l}, k_{l}} / \lambda_{l}}\right) .
\end{aligned}
$$

Then by (S), it follows that $\operatorname{tr} \operatorname{deg}(K: \overline{\mathbb{Q}})=l+1$. From (33) we see that $S-C_{0} \in K$. If $S-C_{0} \in \overline{\mathbb{Q}} \backslash\{0\}$, then there exists a non-zero polynomial 
$A(x) \in \mathbb{Z}[x]$ such that $A\left(S-C_{0}\right)=0$. Hence $\operatorname{tr} \operatorname{deg}(K: \overline{\mathbb{Q}}) \leq l$ and the contradiction obtained proves $\left(\mathrm{S}_{1}\right)$.

REMARK 5.1. If all $\alpha_{j, k} \in \mathbb{Q}(i \sqrt{d})$, then $\left(\mathrm{S}_{1}\right)$ is true by Theorem 1 .

By a similar argument we have

Conjecture $\left(\mathrm{S}_{2}\right)$. Let $P_{1}, \ldots, P_{s}, Q_{1}, \ldots, Q_{s} \in \overline{\mathbb{Q}}[x], r_{1}, \ldots, r_{s} \in \mathbb{Z}$, where for any $1 \leq j \leq s$ the polynomials $P_{j}, Q_{j}$ satisfy the following conditions: $\operatorname{deg} P_{j} \leq \operatorname{deg} Q_{j}-1, Q_{j}\left(r_{j} / 2\right) \neq 0, Q_{j}(n) \neq 0, n=0,1, \ldots$, and

$$
\frac{P_{j}(-x)}{Q_{j}(-x)}=(-1)^{r_{j}} \frac{P_{j}\left(r_{j}+x\right)}{Q_{j}\left(r_{j}+x\right)} .
$$

Then the sum

$$
T=\sum_{n=0}^{\infty}\left(\frac{P_{1}(n)}{Q_{1}(n)}+\cdots+\frac{P_{s}(n)}{Q_{s}(n)}\right)(-1)^{n}
$$

is either a computable algebraic number or transcendental.

Conjecture $\left(\mathrm{S}_{3}\right)$. Let $f: \mathbb{Z} \rightarrow \overline{\mathbb{Q}}$ be periodic with period $q \in \mathbb{N}$. Suppose that $r \in \mathbb{Z}, P(x), Q(x) \in \overline{\mathbb{Q}}[x],\left(Q^{\prime}(q r / 2)\right)^{2}+(Q(q r / 2))^{2} \neq 0, Q(n) \neq 0$, $n=1,2, \ldots$,

$$
\frac{P(-x)}{Q(-x)}= \pm \frac{P(x+q r)}{Q(x+q r)}
$$

and $f$ is an even or odd function according to whether we have the "plus" or "minus" sign in (35). Suppose further that the series

$$
U=\sum_{n=1}^{\infty} \frac{P(n)}{Q(n)} f(n)
$$

converges. Then $U$ is either a computable algebraic number or transcendental.

Conjecture $\left(\mathrm{S}_{4}\right)$. Suppose that $\beta_{1}, \ldots, \beta_{s} \in[0,2)$ are distinct rational numbers, $Q(x), P_{1}(x), \ldots, P_{s}(x) \in \overline{\mathbb{Q}}[x], Q(n) \neq 0, n \in \mathbb{Z}, h(n)=$ $\sum_{j=1}^{s} P_{j}(n) e^{i \pi \beta_{j} n}$, and for $1 \leq j \leq s, \operatorname{deg} P_{j}(x) \leq \operatorname{deg} Q(x)-1$ if $0<\beta_{j}<2$ and $\operatorname{deg} P_{j}(x) \leq \operatorname{deg} Q(x)-2$ if $\beta_{j}=0$. Then the sum

$$
V=\sum_{n=-\infty}^{\infty} \frac{h(n)}{Q(n)}
$$

is either zero or transcendental.

\section{References}

[1] S. D. Adhikari, N. Saradha, T. N. Shorey and R. Tijdeman, Transcendental infinite sums, Indag. Math. (N.S.) 12 (2001), 1-14. 
[2] H. Bateman and A. Erdélyi, Higher Transcendental Functions, Vol. 1, McGraw-Hill, New York, 1953.

[3] P. Bundschuh, Zwei Bemerkungen über transzendente Zahlen, Monatsh. Math. 88 (1979), 293-304.

[4] A. A. Karatsuba, Principles of Analytic Number Theory, Nauka, Moscow, 1975 (in Russian).

[5] G. Molteni, Some arithmetical properties of the generating power series for the sequence $\{\zeta(2 k+1)\}_{k=1}^{\infty}$, Acta Math. Hungar. 90 (2001), 133-140.

[6] M. R. Murty and N. Saradha, Transcendental values of the digamma function, J. Number Theory 125 (2007), 298-318.

[7] Yu. V. Nesterenko, Modular functions and transcendence questions, Mat. Sb. 187 (1996), no. 9, 65-96 (in Russian).

[8] P. Ribenboim, My Numbers, My Friends, Popular Lectures on Number Theory, Springer, Berlin, 2000.

[9] N. Saradha and R. Tijdeman, On the transcendence of infinite sums of values of rational functions, J. London Math. Soc. (3) 67 (2003), 580-592.

[10] R. Tijdeman, On irrationality and transcendency of infinite sums of rational numbers, submitted.

[11] I. M. Vinogradov, An Introduction to the Theory of Numbers, Pergamon Press, London, 1955.

[12] M. Waldschmidt, Open diophantine problems, Moscow Math. J. 4 (2004), 245-305.

Institute for Studies in Theoretical Physics and Mathematics (IPM)

Tehran, Iran

Current address:

Mathematics Department

Faculty of Science

Shahrekord University

P.O. Box 115

Shahrekord, Iran

E-mail: hessamik@ipm.ir

hessamit@gmail.com

Received on 18.9.2006

and in revised form on 4.6.2007 